\title{
P-glycoprotein ABCB1: a major player in drug handling by mammals
}

Piet Borst and Alfred H. Schinkel

Division of Molecular Oncology, The Netherlands Cancer Institute, Amsterdam, The Netherlands.

\begin{abstract}
Mammalian P-glycoproteins are active drug efflux transporters located in the plasma membrane. In the early nineties, we generated knockouts of the three P-glycoprotein genes of mice, the Mdr1a, Mdr1b, and Mdr2 P-glycoproteins, now known as $A b c b 1 a, A b c b 1 b$, and $A b c b 4$, respectively. In the JCI papers that are the subject of this Hindsight, we showed that loss of Mdr1a $(A b c b 1 a)$ had a profound effect on the tissue distribution and especially the brain accumulation of a range of drugs frequently used in humans, including dexamethasone, digoxin, cyclosporin $A$, ondansetron, domperidone, and loperamide. All drugs were shown to be excellent substrates of the murine ABCB1A P-glycoprotein and its human counterpart, the MDR1 P-glycoprotein, ABCB1. We found that the ability of $A B C B 1$ to prevent accumulation of some drugs in the brain is a prerequisite for their clinical use, as absence of the transporter led to severe toxicity or undesired CNS pharmacodynamic effects. Subsequent work has fully confirmed the profound effect of the drug-transporting ABCB1 P-glycoprotein on the pharmacokinetics of drugs in humans. In fact, every new drug is now screened for transport by ABCB1, as this limits oral availability and penetration into sanctuaries protected by $A B C B 1$, such as the brain.
\end{abstract}

\section{Generating knockout mice}

Generating knockout mice in the early 1990s was quite an adventure. The technology had just been developed, and the homologous recombination required to disrupt genes in mouse embryonic stem cells was still extremely inefficient. We were lucky, however, to work in The Netherlands Cancer Institute, where Hein te Riele and Anton Berns discovered in 1992 that gene targeting in embryonic stem cells could be made much more efficient by using isogenic DNA for transfection rather than DNA from a different mouse strain (1). Nevertheless, generating knockout mice remained a risky undertaking.

After protracted lab discussions, Alfred Schinkel, then a senior postdoc in the Borst lab, took the jump. With hindsight, this was the right decision. Although there were indications that drug-transporting P-glycoproteins could be important in drug pharmacokinetics, it was hard to get conclusive data, as the available inhibitors of P-glycoproteins were still toxic and not very

Conflict of interest: The research groups of the authors receive revenue from commercial distribution of some of the mouse strains mentioned in this paper.

Citation for this article: J Clin Invest.

2013;123(10):4131-4133. doi:10.1172/JCI70430. specific. The knockout mice would fill that gap. We reduced the risk of getting scooped by generously explaining to our colleagues elsewhere that we had an edge and would get there first with the expert help of Anton Berns and his knockout crew. Apparently, the competition lost heart, and, in the end, we were the only lab to produce P-glycoprotein knockout mice.

\section{A dead mouse with an unguarded blood-brain barrier}

Before we were able to properly analyze the Mdr1a (Abcb1a) knockout mice, Schinkel received a message from our animal facility that his knockout mice were suddenly dying. When the animal caretakers were asked what they had done, their answer was predictably, "Nothing, we did nothing." It soon emerged, however, that the mice had been sprayed with the pesticide ivermectin to counteract a mite infestation. A toxicity analysis demonstrated that the homozygous $M d r 1 a^{-/-}$mice were 100 -fold more sensitive to ivermectin than their $M d r 1 a^{+/+}$littermates (2). Ivermectin is a strong neurotoxin, but normally it is kept out of the brains of mammals by ABCB1. In the $M d r 1 a^{-/}$mice, about 100 -fold more ivermectin accumulated in the brain, leading to lethal neurotoxicity.
This and subsequent work (3-7) established that a drug-transporting P-glycoprotein is an integral part of the blood-brain barrier (BBB) and essential in keeping large, hydrophobic drugs out of the brain. This conclusion was not directly accepted by scientists working on the BBB. Soon after the ivermectin mishap, Piet Borst was invited to address the European BBB Society as a late-breaking speaker. Before the talk, an established BBB researcher explained why large, amphipathic drugs were unable to pass the BBB, using purely chemical arguments, hydrogen bonds, etc. Borst then came with his disappointing message: it is not the subtle chemistry, but a pump that drives the drug from the apical membrane of the cerebral endothelium back into the circulation. This message was not well received. One discussant shouted in desperation, "Did you verify that every single capillary in the brain contains P-glycoprotein?” Of course we had not, but Borst explained that mice without MDR1A P-glycoprotein were "stone dead" after ivermectin administration, indicating that P-glycoprotein should be an essential component of the entire BBB.

The ABCB1 P-glycoprotein also limits uptake of some drugs from the gut

The presence of ABCB1 in the apical membrane of the intestine made it likely that this protein would also impede the uptake of the substrate drugs. It took time before this was proven and even longer before it became accepted that P-glycoprotein is a major factor in the oral availability of some substrate drugs. In fact, when we showed that the MDR1A P-glycoprotein impedes the uptake of digoxin from the gut (5), it was hard to get this ground-breaking result published. Acceptance improved when we obtained the same result for the anticancer drug paclitaxel. In that case, uptake of the drug is nearly completely prevented by P-glycoprotein, making it necessary to administer the drug intravenously (6). The demonstration that P-glycoprotein limits the oral availability of some drugs as well as their penetration into the brain has led to 


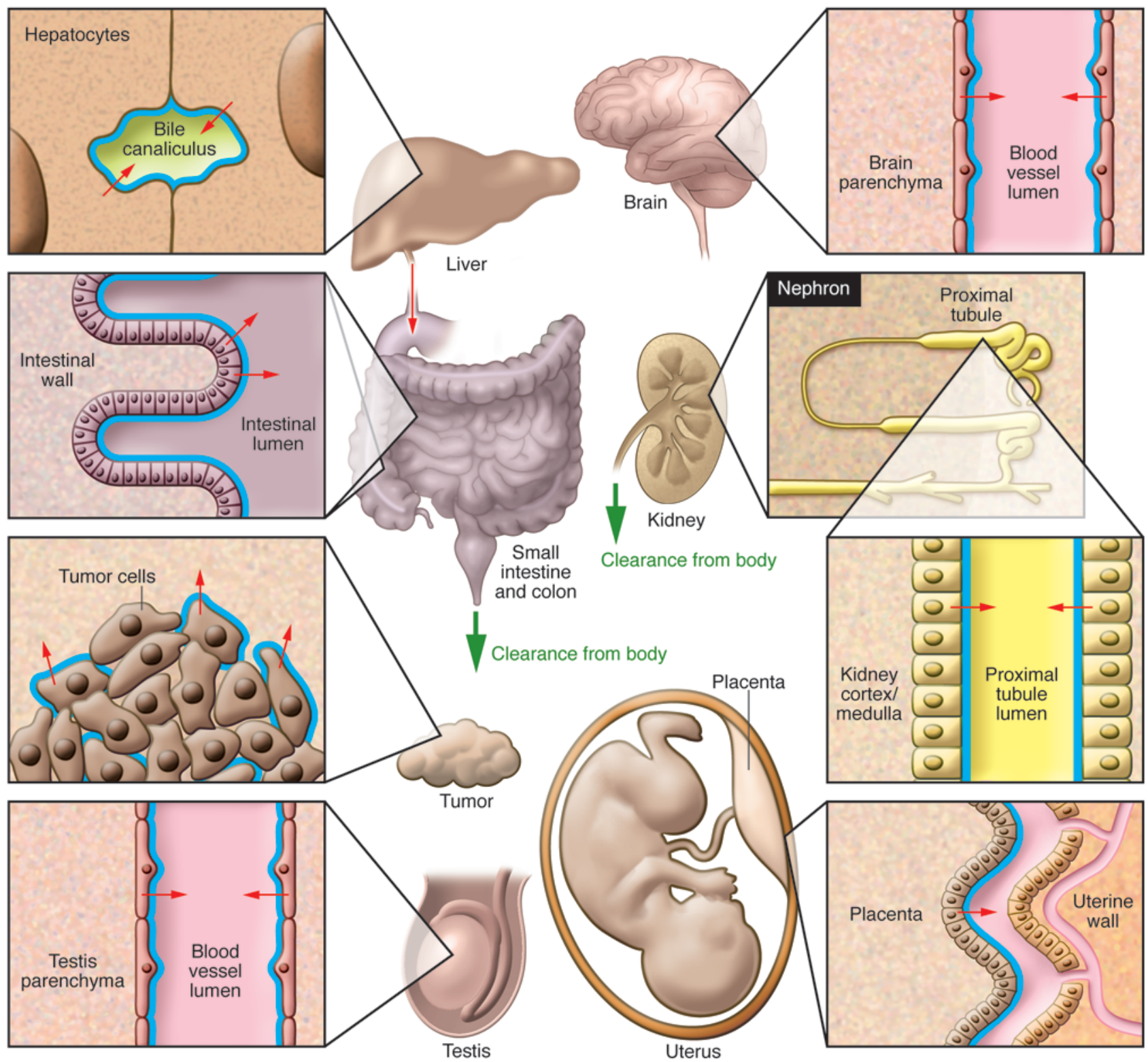

Figure 1

Overview of $A B C B 1$ functional expression throughout the body. Blue lines indicate the location of $A B C B 1$. Small arrows indicate the direction of $A B C B 1-m e d i a t e d$ transport. Bold green arrows indicate net body excretion of $A B C B 1$ substrates. $A B C B 1$ in the BBB and blood-testis and placental fetal-maternal barrier protects brain, testis, and fetus, respectively, from accumulation of $A B C B 1$ substrates. ABCB1 in small and large intestine reduces intestinal uptake (oral availability) and mediates direct intestinal excretion of substrates. ABCB1 in liver and kidney mediates hepatobiliary and renal excretion of substrates. ABCB1 expression in tumor cells can contribute to multidrug resistance.

numerous attempts to eliminate this barrier by using increasingly specific P-glycoprotein inhibitors. P-glycoprotein has also become a buzzword in the pharma industry, as everybody prefers orally available drugs.

\section{Drug-transporting P-glycoprotein today}

As the ABCB1 P-glycoprotein is a highly versatile, if not promiscuous, drug transporter, the number of drugs affected by
P-glycoprotein has exploded. The number of tissues specifically protected by P-glycoprotein has also increased, as illustrated in Figure 1. Whereas the role of P-glycoprotein in pharmacokinetics is large and undisputed, its role in drug resistance of human tumors has turned out to be much smaller than initially anticipated. There is no doubt that modest amounts of P-glycoprotein can completely protect mouse tumors from substrate drugs, such as taxanes or anthra- cyclines, but human tumors seem to use this effective defense mechanism only rarely (8).

\section{The knockout approach has been fruitful in pharmacology}

After our initial success, we made knockouts of many other transporter protein genes and combined some of these in double knockouts and triple knockouts. Schinkel concentrated on P-glycoprotein and Abcg2 and, more recently, on drug importers and P450 
drug-metabolizing enzymes. Borst knocked out MRPs (ABCCs) (9). Disrupting these genes has generated fundamental insight into the roles of these proteins in physiology and pharmacology. Obviously, eliminating the function of a gene in the germline can lead to secondary adjustments. Indeed, in 1994, we observed some upregulation of the second drug-transporting mouse P-glycoprotein gene, $A b c b 1 b(M d r 1 b)$, in the $M d r 1 a$ knockout (2). Thus far, however, these compensatory alterations have not affected the results of our knockout mice in a major way. We have never had reason to regret our entry into the transporter knockout field in 1990, and there is more to come.

\section{Acknowledgments}

We are grateful to the Dutch Cancer Society for their continued financial support of most of the work reviewed here. We also thank all our collaborators for their contributions and, especially, Els Wagenaar for her unrelenting dedication and success in generating the early knockout strains.

Address correspondence to: Piet Borst, Division of Molecular Oncology, The Netherlands Cancer Institute, Plesmanlaan 121, 1066 CX Amsterdam, The Netherlands. Phone: 31.20.512.2880; Fax: 31.20.669.1383; E-mail: p.borst@nki.nl.

1. Te Riele H, Robanus-Maandag EC, Berns A. Highly efficient gene targeting in embryonic stem cells via homologous recombination with isogenic DNA constructs. Proc Natl Acad Sci U S A 1992;89(11):5128-5132.

2. Schinkel AH, et al. Disruption of the mouse $m d r 1$ a P-glycoprotein gene leads to a deficiency in the blood-brain barrier and to increased sensitivity to drugs. Cell. 1994;77(4):491-502.

3. Schinkel AH, Wagenaar E, Van Deemter L, Mol CAAM, Borst P. Absence of the mdr1a P-glycoprotein in mice affects tissue distribution and phar- macokinetics of dexamethasone, digoxin, and cyclosporin A. J Clin Invest. 1995;96(4):1698-1705.

4. Schinkel AH, Wagenaar E, Mol CAAM, van Deemter L. P-glycoprotein in the blood-brain barrier of mice influences the brain penetration and pharmacological activity of many drugs. J Clin Invest. 1996;97(11):2517-2524.

5. Mayer U, et al. Substantial excretion of digoxin via the intestinal mucosa and prevention of long-term digoxin accumulation in the brain by the mdr1a P-glycoprotein. Br J Pharmacol. 1996; 119(5):1038-1044.

6. Sparreboom A, et al. Limited oral bio-availability and active epithelial excretion of paclitaxel (taxol) caused by P-glycoprotein in the intestine. Proc Natl Acad Sci U S A. 1997;94(5):2031-2035.

7. Schinkel AH, et al. Normal viability and altered pharmacokinetics in mice lacking mdr1-type (drug-transporting) P-glycoproteins. Proc Natl Acad Sci U S A. 1997;94(8):4028-4033.

8. Borst P. Cancer drug pan-resistance: pumps, cancer stem cells, quiescence, epithelial to mesenchymal transition, blocked cell death pathways, persisters or what? Open Biol. 2012;2(5):120066.

9. Borst P, Elferink RO. Mammalian ABC transporters in health and disease. Annu Rev Biochem. 2002;71:537-592. 